\title{
The effect of disfluency on mind wandering during text comprehension
}

\author{
Myrthe Faber $^{1}\left(\mathbb{D} \cdot\right.$ Caitlin Mills $^{1} \cdot$ Kristopher Kopp $^{1,2} \cdot$ Sidney D’Mello ${ }^{1,3}$
}

Published online: 6 September 2016

(C) Psychonomic Society, Inc. 2016

\begin{abstract}
When reading, we frequently find ourselves thinking about something other than the text. These attentional lapses, known as mind wandering (MW), are negatively correlated with text comprehension. Previous studies have shown that more syntactically and semantically difficult texts elicit more MW, because textual difficulty impedes the construction of a mental model of the text, which makes it more difficult to suppress off-task thoughts. But is it possible to reduce MW without altering the content of the text itself? We hypothesized that reading a perceptually disfluent text might require more attentional resources, even if the content remained the same, leaving fewer resources available for MW. To test this idea, we manipulated the typefaces (fluent [Arial] or disfluent [Comic Sans]) of two instructional texts on scientific research methods (each about 1,490 words long), and found that MW was less frequent when participants read the disfluent text. There were no comprehension differences between the fluent and disfluent groups. However, we did find an indirect effect of disfluency on comprehension through MW, suggesting that disfluency influences comprehension by enhancing attention. These findings provide insights into how processing difficulty and attention interact during reading comprehension.
\end{abstract}

Myrthe Faber

mfaber@nd.edu

1 Department of Psychology, University of Notre Dame, 224 Haggar Hall, Notre Dame, IN 46556, USA

2 Department of Psychology, Northern Illinois University, Psychology-Computer Science Building, DeKalb, IL 60115, USA

3 Department of Computer Science, University of Notre Dame, 352 Fitzpatrick Hall, Notre Dame, IN 46556, USA
Keywords Attention $\cdot$ Reading $\cdot$ Learning

Attention is critical during reading, because textual information needs to be integrated with an individual's internal representations for comprehension to occur (Smallwood, Fishman, $\&$ Schooler, 2007). Lapses in attention cause a breakdown in this integration, leading to an impoverished representation of information and diminished comprehension (Smallwood, 2013; Smallwood et al., 2007). During these lapses, attention might be directed toward internal self-generated thoughts and feelings. This phenomenon is known as mind wandering (MW) and is estimated to occur $20 \%-40 \%$ of the time during reading (Smallwood et al., 2007).

Previous studies have shown that MW is consistently negatively associated with text comprehension (Feng, D'Mello, \& Graesser, 2013; Smallwood et al., 2007). A reduction in MW would thus benefit comprehension, so it would be useful to identify factors that reduce MW. Several studies have shown that MW is less likely to occur under difficult than under easy conditions in the context of relatively simple sustainedattention tasks (Smallwood, Obonsawin, \& Heim, 2003; Smallwood, Obonsawin, \& Reid, 2003). The idea is that task-related and internal self-generated thoughts (i.e. MW) compete for a limited pool of executive resources that direct attention, so more difficult tasks would be less susceptible to MW because they are less automated and consume more resources (Kane \& McVay, 2012; Kopp \& D’Mello, 2016; Smallwood, 2013; Smallwood \& Schooler, 2006).

However, an opposite effect of difficulty has been reported for more complex tasks. In the context of reading, MW has been found to be more frequent when reading a difficult text than when reading an easier text (Feng et al., 2013; Mills, D’Mello, \& Kopp, 2015). In these studies, difficulty was 
manipulated via textual features such as syntactic complexity and the use of unfamiliar terms. These features target the text base level, but ultimately influence deeper levels of text comprehension by impeding the construction of a situation model (Zwaan \& Radvansky, 1998). When the situation model is impaired, it becomes more difficult to suppress selfgenerated thoughts and feelings, leading to an increase in MW (Feng et al., 2013; Smallwood, 2011).

Instead of targeting difficulty at the text base level, it is also possible to vary difficulty at the surface level, without changing the textual content. For example, perceptual difficulty (also known as disfluency) can be increased by changing the typeface of a text to one that is more difficult to read (e.g., from Arial to Comic Sans; see Alter \& Oppenheimer, 2009b, for an extensive review). Several studies have shown that disfluency can have a positive effect on memory retention in list learning, suggesting that information that is more difficult to decode is encoded better on a surface level (DiemandYauman, Oppenheimer, \& Vaughan, 2011). The additional cognitive burden created by disfluency can thus lead to a desirable outcome, namely deeper processing and better retention.

When it comes to reading, however, researchers have failed to find a consistently significant effect of disfluency on text comprehension (Eitel \& Kühl, 2016; Eitel, Kühl, Scheiter, \& Gerjets, 2014; Lehmann, Goussios, \& Seufert, 2016; Rummer, Schweppe, \& Schwede, 2016; Strukelj, Scheiter, Nyström, \& Holmqvist, 2016). The incongruence between these findings and those for tasks like list learning suggests that disfluency only affects surface-level learning (e.g., list retention), but not the construction of the situation model (e.g., text comprehension).

Does this mean that disfluency does not affect text comprehension at all? We argue that disfluency might indirectly affect comprehension by reducing MW. Specifically, the increased surface-level processing incurred when reading a disfluent text should leave fewer attentional resources available for MW, which in turn should have a positive effect on comprehension.

In line with this hypothesis, we tested two hypotheses on the relationship between perceptual disfluency, MW and text comprehension. First, consistent with studies showing reduced MW for tasks that require more attentional resources (but that do not impede the construction of a mental model), we predicted that an increase in perceptual disfluency should reduce MW. Second, given the preponderance of null effects in the literature (noted above), it was unlikely that we would find a direct effect of perceptual disfluency on text comprehension. However, we expected that reduced MW attributed to disfluency should be related to improved text comprehension, suggesting that MW mediates the relationship between perceptual disfluency and comprehension. By analyzing the influence of disfluency on MW, we hoped to provide an explanation for the puzzling lack of effect of disfluency on text comprehension.

\section{Method}

\section{Participants}

A total of 207 Amazon Mechanical Turk (AMT) workers (U.S. residents only) completed this experiment. Participation was voluntary, and participants received $\$ 2$ in compensation. AMT can be used to collect reliable and valid experimental data (Mason \& Suri, 2012), but in contrast with a lab study, experimenters have less control over the experimental settings, and participants' ages and education levels may vary more than when sampling from a student population. However, given that online reading and learning is ubiquitous nowadays, sampling from a heterogeneous population in a less-controlled setting increased the ecological validity of the study and the authenticity of the learning experience.

The sample size was determined on the basis of the minimum detectable effect size (MDES) for the main effect of disfluency on MW. An MDES calculation (Raudenbush, 2011) using a two-tailed alpha of .05 and an observable power of .8 suggested that a total sample size of around 205 participants would be necessary to detect an effect of 0.4 sigma (Cohen's $d$ ), which is within the small-to-medium range. A large sample was also needed for mediation analyses.

\section{Design}

The experiment utilized a between-subjects design. Participants read one of two texts in either Arial typeface, for the fluent condition, or Comic Sans typeface, for the disfluent condition (Fig. 1; the choices of typefaces were based on Diemand-Yauman et al., 2011). The assignment of conditions was random, and the text assignment was counterbalanced across participants.

\section{Materials}

Texts Two texts on scientific research methods were taken from the electronic textbook that accompanies the educational game Operation ARA! (Halpern et al., 2012). These texts have been used in previous online reading studies (Mills et al., 2015; Phillips, Mills, D’Mello, \& Risko, 2016). Research methods topics were used because the texts were educational, relatively unfamiliar to the average reader, and useful to a diverse population.

The text on dependent variables was 104 sentences long (1,490 words), and the text on causal inference was 108 sentences long (1,491 words). Both texts were at a moderate, 9th-grade level in terms of textual difficulty on the Flesch- 
Fig. 1 Illustration of the typefaces used.
Fluent (Arial, 20 point)

One common misunderstanding is that any study that involves an existing group (e.g., females and males, attractive and less attractive people) must always be correlational.

\section{Disfluent (Comic Sans MS italicized, 16 point, Gray)}

Kincaid scale (Klare, 1974). Each text was presented one sentence at a time, and participants used the space bar to move to the next sentence. They could not move back to a previously read sentence.

Subjective perceptions Participants completed a five-item questionnaire (Phillips et al., 2016) about their subjective experience after reading. The questionnaire measured effort ("I put a lot of effort into this"), interest ("I would describe this activity as very interesting"), and value ("I believe doing this activity could be beneficial to me"), to ensure that motivation was equivalent across conditions. Perceived difficulty ("I believe the reading level of the text was very difficult") and competence ("I think I understood this text very well") were also assessed, to test whether the disfluency was consciously perceived. Each of these items was rated on a 6-point scale from 1 (strongly disagree) to 6 (strongly agree).

Mind-wandering probes Participants were instructed to report MW by responding to auditory thought probes (a beep) throughout reading. A standard description of MW (based on Smallwood \& Schooler, 2006) was provided before the reading task: "Sometimes when you are reading, you may suddenly realize that you are not thinking about what it is that you are reading. We call this 'zoning out' about thoughts unrelated to the content of what it is we are reading." Participants were instructed to indicate whether they were or were not mind wandering at the time of the probe by pressing the "y" or the " $n$ " key, respectively. Probes were placed on nine pseudorandom pages throughout each text and would never occur within the first or the last ten sentences, or within five sentences of each other. The probes were triggered when the participant pressed the space bar to move on to the next sentence, so the per-sentence reading times were not influenced by the probe responses.

Comprehension assessment Comprehension was assessed on two levels: text-level questions focused on factual or surface-level characteristics of the text (e.g., "the passage says people who ___ do not fall for unsupported claims?"), whereas inference-level questions required participants to draw inferences or apply their knowledge to a novel example (e.g., "[In this novel example], depression is the variable, and art therapy is the __ variable"). Both question types used a four-alternative multiple-choice format.

Before the reading phase, participants completed a pretest about research methods. This test only included inferencelevel questions, because surface-level questions cannot be answered without having read the text. Depending on the text, this test consisted of six questions about dependent variables or four questions about causal claims. After reading the target text, participants completed a posttest, which consisted of 12 new items for the dependent-variable text (six inference-level questions, six text-level questions) or 12 new items for the causal-claims text (three inference-level, nine text-level). The numbers of questions differed across texts due to the availability of validated items.

\section{Procedure}

Participants received a brief description of the study and were given the option not to volunteer before providing electronic consent. They then completed the pretest questions. Next, they received instructions on how to respond to the MW probes, after which they began the reading task (which included the MW probes). After reading the text, participants completed the subjective perception 
questionnaire and comprehension assessment. On completion, they were fully debriefed.

\section{Data treatment}

Three participants (1.4\%) were removed due to noncompliance (i.e. reading times under $1.5 \mathrm{~min}$; the average reading time was $5.3 \min [S D=2.1]$ ). All dependent variables were $z-$ score-standardized per text for the statistical analyses (unless otherwise noted); unstandardized data are reported for descriptive purposes.

\section{Results}

\section{Subjective perceptions}

The fluency manipulation did not lead to significant differences (at a two-tailed $p<.05$ for this and all subsequent analyses) in perceived difficulty (fluent: $M=2.29, S D=$ 1.26; disfluent: $M=2.09, S D=1.13$ ), effort (fluent: $M=$ 5.16, $S D=0.94$; disfluent: $M=5.27, S D=0.91$ ), or selfrated competence (fluent: $M=4.41, S D=1.17$; disfluent: $M$ $=4.42, S D=0.99$ ), nor did it affect the interest in (fluent: $M=$ 4.10, $S D=1.42$; disfluent: $M=4.20, S D=1.40$ ) or the value of (fluent: $M=4.02, S D=1.50$; disfluent: $M=4.05, S D=$ 1.30) the activity. These results indicate that fluency did not affect the participants' subjective perceptions of the reading task, which is what was expected.

\section{Mind wandering}

We hypothesized that disfluency would lead to lower MW. Due to the nonnormal distribution of standardized MW reports, we used a nonparametric test to analyze these data. A Mann-Whitney $U$ test revealed that the disfluent condition elicited significantly less MW than did the fluent condition (fluent: $M=27.8 \%, S D=28.4 \%$; disfluent: $M=21.0 \%, S D$ $=28.6 \% ; U=4,204.5, p=.017, d=-0.34$ sigma). We also conducted a Poisson regression on the raw MW counts as an additional, confirmatory analysis, which yielded similar results $(p=.017$, null deviance $=616.25$, residual deviance $=$ 600.00 , controlling for text). On average, participants mind wandered $6.8 \%$ less while reading a text in a disfluent typeface, which is consistent with our predictions.

\section{Comprehension}

We observed a trend (albeit nonsignificant) suggesting that text-level comprehension scores were higher for disfluent text (fluent: $M=70.6 \%, S D=23.7 \%$; disfluent: $M=75.5 \%, S D=$ $20.4 \%), F(1,202)=2.28, p=.133, d=0.21$ sigma. Inferencelevel comprehension scores did not differ significantly between conditions (fluent: $M=46.4 \%, S D=23.7 \%$; disfluent $M=49.3 \%, S D=25.8 \%), F(1,202)=0.60, p=.440, d=0.11$ sigma.

\section{Effect of MW on comprehension}

We tested whether the relationship between disfluency and comprehension was mediated by MW. Note that according to contemporary mediation procedures, it is not necessary to observe a significant direct effect of disfluency on comprehension to establish an indirect effect through MW (see Zhao, Lynch, \& Chen, 2010, for a discussion). Indirect effects were computed using the "mediation" package in R (Tingley, Yamamoto, Hirose, Keele, \& Imai, 2014). We specified two models: a mediator model (Poisson regression of MW counts on disfluency) and an outcome-variable model (linear regression of comprehension scores on disfluency and MW). Both models used nonnormalized MW counts, but text was added as a covariate. To control for the fact that longer reading times and prior knowledge are related to comprehension, pretest scores and reading times were added as covariates ${ }^{1}$ to the appropriate outcome variable models. We then obtained causal estimates for the indirect effect of MW over 10,000 quasiBayesian Monte Carlo simulations. We found that the relationship between disfluency and text- and inference-level comprehension was indeed mediated by MW (Table 1), which supports the idea that disfluency positively affects comprehension by reducing MW.

\section{Discussion}

The aim of this study was to investigate the effect of perceptual processing difficulty on mind wandering during text comprehension. We hypothesized that the added perceptual processing required for reading disfluent text would leave fewer cognitive resources available for MW. As hypothesized, we found that MW rates were lower for perceptually disfluent texts with identical content to the fluent texts. Furthermore, we hypothesized

\footnotetext{
${ }^{1}$ An important assumption in mediation analysis is that the relationships between condition, mediator, and outcome are not confounded by other variables. MW was not significantly correlated with reading time (Spearman's $\rho=-.07, p=.30$ ) or prior knowledge (Spearman's $\rho=$ $-.09, p=.19)$. Both reading time and pretest scores were correlated with text-level comprehension $(r=.25, p<.001$, and $r=.16, p=.019$, respectively). Inference-level comprehension was positively correlated with prior knowledge (i.e. pretest scores, $r=.21, p=.003$ ) but not with reading time $(r=.016, p=.816)$. We therefore included covariates in the appropriate models (reading time and prior knowledge for text-level comprehension, prior knowledge for inference-level comprehension), which is a common way to address this issue (Preacher, 2015). Nevertheless, we would point out that the same analyses without covariates yielded similar results (text level: estimate $=.0650$, lower confidence interval $[\mathrm{CI}]=$ .0103 , upper $\mathrm{CI}=.1372, p=.02$; inference level: estimate $=.0434$, lower $\mathrm{CI}=.0040$, upper $\mathrm{CI}=.1024, p=.02$ ).
} 
Table 1 Estimates for the indirect effect of MW on comprehension as an effect of disfluency

\begin{tabular}{lllll}
\hline & Mean & Lower CI & Upper CI & $p$-value \\
\hline Text level & .0594 & .0097 & .1251 & .01 \\
Inference level & .0411 & .0029 & .0966 & .03 \\
\hline
\end{tabular}

The reported CIs are $95 \%$ confidence intervals.

that a decrease in MW due to disfluency would affect text comprehension. Our results indicated that MW mediated the relationship between disfluency and comprehension, suggesting that disfluency has an indirect positive effect on comprehension, by increasing the attentional resources directed to the reading task. Simply put, perceptual disfluency acts as a "desirable difficulty" (cf. Diemand-Yauman et al., 2011) by reducing MW during reading.

The fact that we did not observe a significant direct effect of disfluency on text comprehension is in line with other recent findings (Eitel \& Kühl, 2016; Eitel et al., 2014; Lehmann et al., 2016; Rummer et al., 2016; Strukelj et al., 2016). However, we have shown that disfluency has an indirect effect on text comprehension by reducing MW, thereby elucidating one mechanism by which disfluency influences the reading process. An open question is why studies (including ours) have failed to observe a direct effect of disfluency on comprehension.

One possibility is that although the processing load engendered by disfluency can reduce MW, disfluency itself does not contribute to the construction of a mental model of the text. It can therefore be considered to impose an extraneous cognitive load (see Paas, Renkl, \& Sweller, 2004), which might act as a competing mediator, thereby precluding a direct effect.

Recent work has also suggested that learner characteristics such as need for cognition (Cacioppo \& Petty, 1982) might moderate disfluency effects (Kühl, Eitel, Damnik, \& Körndle, 2014; Kühl, Eitel, Scheiter, \& Gerjets, 2014). A participant's willingness to put effort into learning is likely to affect whether she or he benefits from the increase in processing load. Future research could further explore the effect of individual differences on the relationship between disfluency, MW, and comprehension.

It is important to note that participants did not subjectively perceive reading disfluent text as more difficult or effortful, nor did it affect their ratings of self-competence. This suggests that (as intended) the disfluency manipulation was subtle enough not to be consciously appraised as being a hindrance to comprehension. ${ }^{2}$ Indeed, alternative, content-oriented manipulations of textual difficulty (as opposed to content-free

\footnotetext{
${ }^{2}$ Overall, self-reported difficulty did not significantly predict MW (Spearman's $\rho=.069, p=.328$ ). A trend suggested that (as expected) self-reported difficulty is negatively related to text-level comprehension $(r=-.133, p=.057)$, but not to inference-level comprehension $(r=-.030$, $p=.668)$.
}

manipulations of processing difficulty) are consciously appraised as such and have been shown to increase MW (Feng et al., 2013; Mills et al., 2015). Our study suggests that textual difficulty and perceptual disfluency have opposite effects on MW, supporting the idea that both influence cognitive processing in different ways (Alter \& Oppenheimer, 2009b; Diemand-Yauman et al., 2011). Together, these findings indicate that disfluency can reduce MW without participants being aware of the extra processing cost, and can lead to effects opposite from those of more content-oriented manipulations of text difficulty.

It is also currently unknown whether disfluency interacts with other types of difficulty. For instance, performance might degrade if the cognitive load imposed by disfluency and other task demands (e.g., textual difficulty) were too high (Paas et al., 2004). Further research, including replications with different texts and/or tasks, will be necessary to determine the boundary conditions of the effect of disfluency on MW and comprehension.

Like all research, our study is not without limitations. These findings - and in fact, all studies that rely on self-reported MW-rest upon the assumption that participants report MW accurately and honestly. Disfluency tends to be associated with less self-disclosure of unfavorable information (Alter \& Oppenheimer, 2009a), so the lower MW rates for the disfluent condition might be associated with a decrease in participants' willingness to admit to MW. However, this alternate explanation does not effectively explain the negative relationship between (admitting to) MW and comprehension. Nevertheless, this issue awaits future research with more objective measures of MW.

In conclusion, we have shown that attention is one of the mechanisms affected by disfluency during reading. Although we acknowledge that disfluency might also affect other cognitive mechanisms, we argue that this finding is important to the fields of cognitive and educational psychology, because it suggests that a simple change in typeface can reduce mind wandering, which is negatively associated with text comprehension. This finding is therefore an important step toward a better understanding of how text characteristics and attention interact during reading comprehension.

Author note This research was supported by the National Science Foundation (NSF; Grant Nos. DRL 1235958 and IIS 1523091). Any opinions, findings and conclusions, or recommendations expressed in this study are those of the authors and do not necessarily reflect the views of the NSF.

\section{References}

Alter, A. L., \& Oppenheimer, D. M. (2009a). Suppressing secrecy through metacognitive ease: Cognitive fluency encourages self-disclosure. Psychological Science, 20, 1414-1420. doi:10.1111/j.14679280.2009.02461.x 
Alter, A. L., \& Oppenheimer, D. M. (2009b). Uniting the tribes of fluency to form a metacognitive nation. Personality and Social Psychology Review, 13, 219-235. doi:10.1177/1088868309341564

Cacioppo, J. T., \& Petty, R. E. (1982). The need for cognition. Journal of Personality and Social Psychology, 42, 116-131. doi:10.1037/0022-3514.42.1.116

Diemand-Yauman, C., Oppenheimer, D. M., \& Vaughan, E. B. (2011). Fortune favors the bold (and the Italicized): Effects of disfluency on educational outcomes. Cognition, 118, 111-115. doi:10.1016/j. cognition.2010.09.012

Eitel, A., \& Kühl, T. (2016). Effects of disfluency and test expectancy on learning with text. Metacognition and Learning, 11, 107-121. doi:10.1007/s11409-015-9145-3

Eitel, A., Kühl, T., Scheiter, K., \& Gerjets, P. (2014). Disfluency meets cognitive load in multimedia learning: Does harder-to-read mean better-to-understand? Applied Cognitive Psychology, 28, 488-501. doi:10.1002/acp.3004

Feng, S., D’Mello, S., \& Graesser, A. C. (2013). Mind wandering while reading easy and difficult texts. Psychonomic Bulletin \& Review, 20, 586-592. doi:10.3758/s13423-012-0367-y

Halpern, D. F., Millis, K., Graesser, A. C., Butler, H., Forsyth, C., \& Cai, Z. (2012). Operation ARA: A computerized learning game that teaches critical thinking and scientific reasoning. Thinking Skills and Creativity, 7, 93-100. doi:10.1016/j.tsc.2012.03.006

Kane, M. J., \& McVay, J. C. (2012). What mind wandering reveals about executive-control abilities and failures. Current Directions in Psychological Science, 21, 348-354. doi:10.1177/0963721412454875

Klare, G. R. (1974). Assessing readability. Reading Research Quarterly, 10, 62-102. doi:10.2307/747086

Kopp, K., \& D'Mello, S. (2016). The impact of modality on mind wandering during comprehension. Applied Cognitive Psychology, 30, 29-40. doi:10.1002/acp.3163

Kühl, T., Eitel, A., Damnik, G., \& Körndle, H. (2014). The impact of disfluency, pacing, and students' need for cognition on learning with multimedia. Computers in Human Behavior, 35, 189-198. doi:10.1016/j.chb.2014.03.004

Kühl, T., Eitel, A., Scheiter, K., \& Gerjets, P. (2014). A call for an unbiased search for moderators in disfluency research: Reply to Oppenheimer and Alter (2014). Applied Cognitive Psychology, 28, 805-806. doi:10.1002/acp.3030

Lehmann, J., Goussios, C., \& Seufert, T. (2016). Working memory capacity and disfluency effect: An aptitude-treatment-interaction study. Metacognition and Learning, 11, 89-105. doi:10.1007/s11409-0159149-z

Mason, W., \& Suri, S. (2012). Conducting behavioral research on Amazon's Mechanical Turk. Behavior Research Methods, 44, 123. doi:10.3758/s13428-011-0124-6

Mills, C., D’Mello, S. K., \& Kopp, K. (2015). The influence of consequence value and text difficulty on affect, attention, and learning while reading instructional texts. Learning and Instruction, 40, 9 20. doi:10.1016/j.learninstruc.2015.07.003
Paas, F., Renkl, A., \& Sweller, J. (2004). Cognitive load theory: Instructional implications of the interaction between information structures and cognitive architecture. Learning and Instruction, 32, 1-8. doi:10.1023/B:TRUC.0000021806.17516.d0

Phillips, N., Mills, C., D’Mello, S. K., \& Risko, E. (2016). On the influence of re-reading on mind wandering. Quarterly Journal of Experimental Psychology. doi:10.1080/17470218.2015.1107109. Advance online publication.

Preacher, K. J. (2015). Advances in mediation analysis: A survey and synthesis of new developments. Annual Review of Psychology, 66, 825-852. doi:10.1146/annurev-psych-010814-015258

Raudenbush, S. W. (2011). Optimal design software for multi-level and longitudinal research (Version 3.01). Retrieved from www. wtgrantfoundation.org

Rummer, R., Schweppe, J., \& Schwede, A. (2016). Fortune is fickle: Null-effects of disfluency on learning outcomes. Metacognition and Learning, 11, 57-70. doi:10.1007/s11409-015-9151-5

Smallwood, J. (2011). Mind-wandering while reading: Attentional decoupling, mindless reading and the cascade model of inattention. Linguistics and Language Compass, 5, 63-77. doi:10.1111/j.1749818X.2010.00263.X

Smallwood, J. (2013). Distinguishing how from why the mind wanders: A process-occurrence framework for self-generated mental activity. Psychological Bulletin, 139, 519-535. doi:10.1037/a0030010

Smallwood, J., Fishman, D. J., \& Schooler, J. W. (2007). Counting the cost of an absent mind: Mind wandering as an underrecognized influence on educational performance. Psychonomic Bulletin \& Review, 14, 230-236. doi:10.3758/BF03194057

Smallwood, J., Obonsawin, M., \& Heim, D. (2003). Task unrelated thought: The role of distributed processing. Consciousness and Cognition, 12, 169-189. doi:10.1016/S1053-8100(02)00003-X

Smallwood, J., Obonsawin, M., \& Reid, H. (2003). The effects of block duration and task demands on the experience of task unrelated thought. Imagination, Cognition and Personality, 22, 13-31. doi:10.2190/TBML-N8JN-W5YB-4L9R

Smallwood, J., \& Schooler, J. W. (2006). The restless mind. Psychological Bulletin, 132, 946-958. doi:10.1037/00332909.132.6.946

Strukelj, A., Scheiter, K., Nyström, M., \& Holmqvist, K. (2016). Exploring the lack of a disfluency effect: Evidence from eye movements. Metacognition and Learning, 11, 71-88. doi:10.1007/s11409-0159146-2

Tingley, D., Yamamoto, T., Hirose, K., Keele, L., \& Imai, K. (2014). Mediation: R package for causal mediation analysis. Journal of Statistical Software, 59, 1-38. doi:10.18637/jss.v059.105

Zhao, X., Lynch, J. G., Jr., \& Chen, Q. (2010). Reconsidering Baron and Kenny: Myths and truths about mediation analysis. Journal of Consumer Research, 37, 197-206. doi:10.1086/651257

Zwaan, R. A., \& Radvansky, G. A. (1998). Situation models in language comprehension and memory. Psychological Bulletin, 123, 162-182. doi:10.1037/0033-2909.123.2.162 